\title{
MiR-135a Protects Vascular Endothelial Cells Against Ventilator-Induced Lung Injury by Inhibiting PHLPP2 to Activate PI3K/Akt Pathway
}

\author{
Xiaodi Yan ${ }^{a}$ Wenqian Lia Liye Yang ${ }^{a}$ Wenwen Dong ${ }^{b}$ Wei Chen ${ }^{a}$ Yanfei Mao ${ }^{b}$ \\ Pingbo Xuc Dongjie Lid Hongbin Yuan ${ }^{\mathrm{a}}$ Yonghua Lia \begin{abstract}
bDepartment of Anesthesiology and Surgical Intensive Care Unit, Xinhua Hospital, Shanghai Jiaotong University School of Medicine, Shanghai, 'Department of Anesthesiology, Fudan University Shanghai Cancer Center, Department of Oncology, Shanghai Medical College, Fudan University, Shanghai, ${ }^{\mathrm{d}}$ Department of Pharmacy, Shanghai Tenth People's Hospital, Tongji University School of Medicine, Shanghai, China
\end{abstract} \\ aDepartment of Anesthesiology, Changzheng Hospital, Second Military Medical University, Shanghai,
}

\section{Key Words}

Mir-135a • Ventilator-induced lung injury • Human umbilical vein endothelial cells • PI3K • PHLPP

\begin{abstract}
Background/Aims: Loss of endothelial barrier function plays an important role in the development of ventilator-induced lung injury (VILI). This study aimed to investigate the effects of miR135a on VILI in a model of mechanical stretch (MS)-induced human umbilical vein endothelial cell (HUVEC) injury. Methods: HUVECs were randomly assigned to 7 groups: blank, negative control (NC), NC+MS, miR135a over-expression (mi-miR135a), mimiR135a+MS, miR135a silencing (si-miR135a) and si-miR135a+MS groups. MS was induced by subjecting cells to cyclic stretch at $20 \%$ stretch for $4 \mathrm{~h}$. After $24 \mathrm{~h}$, levels of reactive oxygen species (ROS) were measured by DCFH-DA fluorescence intensity. Apoptosis was measured using annexin V-FITC/propidium iodide assay with flow cytometry. Inflammatory cytokine levels were determined by ELISA. Barrier integrity was determined using FITC-conjugated dextran assay. Expression levels of PI3K, p-PI3K, Akt, p-Akt, Bcl-2 and Bax were examined using western blotting. The interaction between miR135a and PHLPP2 was evaluated by dualluciferase reporter assay. Results: Our results showed that MS reduced cell numbers, increased the number of apoptotic cells, increased ROS, barrier dysfunction and inflammatory cytokines in HUVECs, and reduced p-PI3K and p-Akt expression; silencing of miR135a worsened MSinduced HUVEC injury. However, miR135a over-expression protected HUVECs against MSinduced increases in apoptotic cells, ROS, barrier dysfunction and inflammatory cytokines, Xiaodi Yan, Wenqian Li and Liye Yang contributed equally to this work.

Dr. Yong-Hua Li,

Dr. Hong-Bin Yuan

And Dr. Dong-Jie Li

Department of Anesthesiology, Changzheng Hospital, Second Military Medical University,

415 Fengyang Road, Shanghai, 200003 (China)

Email liyonghua1207@smmu.edu.cn, jfjczyy@aliyun.com, Idjbio@126.com
\end{abstract}


which were accompanied by activation of the PI3K/Akt signaling pathway. Simultaneous silencing of miR135a and PHLPP2 partially salvaged the effects of miR135a silencing, and miR135a was found to interact with PHLPP2. Conclusion: miR135a may protect HUVECs from MS-induced injury by inhibiting PHLPP2 to activate PI3k/Akt signaling pathway.

(C) 2018 The Author(s)

Published by S. Karger AG, Basel

\section{Introduction}

Mechanical ventilation (MV) is part of basic life support during general anesthesia as well as in critically ill patients with and without respiratory failure. However, MV itself has the potential to cause or worsen lung injury, which is known as ventilator-induced lung injury (VILI) [1]. Acute respiratory distress syndrome (ARDS) accounts for approximately one-third of intensive care unit admissions [2] and nearly all patients with ARDS require MV. Therefore, ARDS patients are usually at risk for VILI, and VILI has been definitively shown to contribute to mortality in patients with ARDS [3]. Although the precise pathogenic mechanism of VILI has not been completely clarified, several mechanisms have been proposed. Currently, inflammation and oxidative stress are accepted as important mechanisms in the pathogenesis of VILI, and thus inhibition of inflammation and/or oxidation is used for protection against VILI [4]. At the molecular level, G-protein-coupled receptors, stretchactivated ion channels, receptor tyrosine kinases, and other molecules are also involved in the pathogenesis of VILI $[4,5]$. Increasing evidence indicates that loss of endothelial barrier function plays an important role in the development of VILI [6]. Mechanical stress may induce post-translational modifications to affect several important signaling pathways involved in VILI responses of the endothelium [6].

MicroRNAs (miRNAs) are an evolutionarily conserved group of small non-coding RNA molecules (containing about 22 nucleotides) and play important roles in a variety of physiological and pathological processes [7]. Growing evidence indicates that dysregulation of miRNAs is closely associated with the pathogenesis of diseases including lung diseases [8]. Vaporidi et al. investigated lung microRNA profiling in a mouse model of VILI and found that the expression of 65 miRNAs changed more than two-fold upon high tidal volume ventilation (HTVV; $40 \mathrm{~mL} / \mathrm{kg}$ ); antagonism of miR-21, an miRNA highly upregulated by HTVV, was able to preserve lung compliance and reduce alveolar-arterial oxygen difference and protein level in bronchoalveolar lavage [9]. In recent years, miR-135a has been extensively studied due to its controversial role in cancers. Mao et al. found that miR-135a was able to enhance cellular proliferation through regulation of $\mathrm{PH}$ domain leucine-rich repeat protein phosphatase (PHLPP) 2 and forkhead box 0 (FOXO) 1 in human bladder cancer [10]. In addition, miR$135 \mathrm{a}$ is related to myocardial depression in sepsis [11] and anxiety-like behavior in the amygdala [12]. However, the role of miR-135a in VILI remains unclear.

In this study, the role of miR-135a in VILI and the relationship between miR-135a and PHLPP2 were investigated using MS in human umbilical vein endothelial cells (HUVECs).

\section{Materials and Methods}

\section{Cell culture and groups}

HUVECs were maintained in Dulbecco's modified Eagle's medium containing $10 \%$ fetal bovine serum, $1 \%$ streptomycin, and penicillin at $37^{\circ} \mathrm{C}$ with $5 \% \mathrm{CO}_{2}$ as described previously [13]. Adherent cells were characterized by their cobblestone morphology. Trypan blue staining showed higher than $95 \%$ cell viability. HUVECs were divided into 7 groups: blank control, negative control (NC), NC+mechanical stretch (MS), miR-135a over-expression (mi-miR-135a), mi-miR-135a+MS, miR-135a silencing (si-miR-135a) and si-miR-135a+MS groups. After various treatments, HUVECs (spindle-shaped) were observed under light microscopy and representative photographs were captured. HUVECs were counted in at least three fields for each group by two investigators blind to the study group, and the mean was calculated. 


\section{Cellular Physiology Cell Physiol Biochem 2018;48:1245-1258

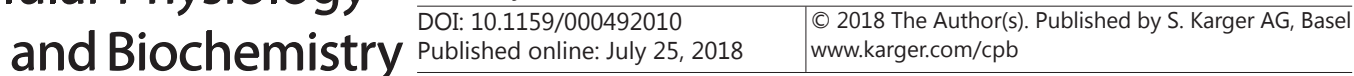

Yan et al.: MiR-135a Reduces Lung Injury

miRNA transfection and PHLPP2 silencing

HUVECs in the logarithmic growth phase were digested with trypsin and then counted. A singlecell suspension was prepared and cells were seeded into 6-well plates at a density of $5 \times 10^{5}$ cells $/ \mathrm{mL}(1$ $\mathrm{mL} /$ well), followed by incubation at $37^{\circ} \mathrm{C}$ with $5 \% \mathrm{CO}_{2}$. HUVECs were grown to $70-80 \%$ confluence and transfected.

Cells were divided into blank control, NC, si-miR-135a and mi-miR-135a groups. In the blank control group, cells were maintained in serum-free Opti-mem ${ }^{\circledR}$ I (Gibco ${ }^{\circledR}$, Waltham, MA, USA) medium $(250 \mu \mathrm{L})$ without other treatment [14]. In the $\mathrm{NC}$ group, $5 \mu \mathrm{L}$ negative control (about $100 \mathrm{pmol}$; 5'-CAGUACUUUUGUGUAGUACAA-3') was added to $245 \mu \mathrm{L}$ serum-free Opti-mem medium; in the si-miR135a group, $5 \mu \mathrm{L}$ miR-135a inhibitor ( $\sim 100$ pmol; 5'-UCACAUAGGAAUAAAAAGCCAUA-3') was added to $245 \mu \mathrm{L}$ serum-free Opti-mem medium; in the mi-miR-135a group, $5 \mu \mathrm{L}$ miR-135a mimic ( 100 pmol; 5'-UAUGGCUUUUUAUUCCUAUGUGA-3') was added to $245 \mu \mathrm{L}$ serum-free Opti-mem medium [15]. Cells were transfected in the presence of Lipofectamine ${ }^{\circledR} 2000$ (Thermo Fisher Scientific, Carlsbad, CA, USA) at $37^{\circ} \mathrm{C}$ for $6 \mathrm{~h}$. Then, the medium was removed and refreshed with normal medium. After $48 \mathrm{~h}$, cells were harvested for experiments.

To investigate the role of PHLPP2 in MS-induced cell injury, PHLPP2 expression was silenced in HUVECs (sense: GACCUCUUCAGAUCGUUUAdTdT; anti-sense: UAAACGAUCUGAAGAGGUCdTdT). Western blotting and real-time PCR (Bio-Rad Laboratories, Hercules, CA, USA) were performed to confirm the silencing efficiency, and apoptotic cells following MS were detected after silencing of miR-135a and/or PHLPP2.

Real-time PCR

To investigate the efficiency of silencing and over-expression, real-time PCR was used to detect miR-135a and PHLPP2 expression. Total RNA was extracted from HUVECs with TRIzol $®$ reagent (Invitrogen, Waltham, MA, USA) [16]. cDNA was generated from total RNA using a cDNA reverse transcription kit (Fermentas, Burlington, NC, USA) with specific primers for miR-135a (5' ACACTCCAGCTGGGTATGGCTTTTTATTCCT 3' [forward], 5' CTCAACTGGTGTCGTGGAGTCGGCAATTCAGTTGAGTCACAT 3' [reverse]), small nuclear RNA U6 (5' CTCGCTTCGGCAGCACA 3' [forward], 5' AACGCTTCACGAATTTGCGT 3' [reverse]) and PHLPP2 (5' ATGGAGCAGACACTACCACTG 3' [forward], 5' GCAAAGGACGAGATGTAAGTCA 3' [reverse]) (Shanghai Sangon Biotech Co., Ltd., Shanghai, China). Quantitative real-time PCR was carried out using SYBR green (Thermo Fisher) as the detection dye and the MiniOpticon real-time PCR detection system (Bio-Rad Laboratories). The primer sequences were designed based on the cDNA sequences in GenBank. The reaction mixture included $2.0 \mu \mathrm{L}$ cDNA, $0.2 \mu \mathrm{mol} / \mathrm{L}$ each paired primer, $200 \mu \mathrm{mol} / \mathrm{L}$ deoxynucleotide triphosphates, $1 \mathrm{U}$ Taq DNA polymerase (Qiagen, Beijing, China) and $1 \times$ PCR buffer. The annealing temperature was set at $58-61^{\circ} \mathrm{C}$ and amplification was set at 40 cycles. The temperature range to detect the melting temperature of the PCR product was set at $60-95^{\circ} \mathrm{C}$. The housekeeping gene GAPDH was used as an internal control for sample loading and normalization. To determine the relative quantitation of gene expression for both target and

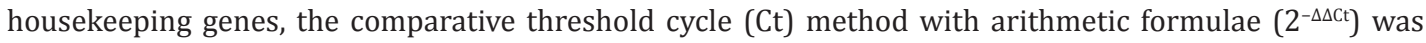
used as described previously [17]. Messenger RNA levels were normalized relative to the housekeeping gene.

\section{MS-induced HUVEC injury}

In the MS groups, cells were subjected to MS to induce injury. In brief, HUVECs were plated onto collagen I-coated Bioflex culture plates (FlexCell International, Burlington, NC, USA) and grown to confluence. After $24 \mathrm{~h}$, HUVECs were subjected to cyclic stretch as previously described [18] using the Flexcell FX-5000 tension system (FlexCell International). The pattern of cyclic stretch was set at a frequency of 30 cycles $/ \mathrm{min}$, with a stretch/relaxation relation of $1 / 1$. Cyclic stretch was conducted at $20 \%$. Unstretched control HUVECs were placed in the same incubator containing $5 \% \mathrm{CO}_{2}$. Cyclic stretch was maintained for $4 \mathrm{~h}$, and then cells were harvested and cultured for $24 \mathrm{~h}$.

Flow cytometry of apoptotic cells

At $24 \mathrm{~h}$ after MS-induced cell injury, cell apoptosis was analyzed with the Annexin V-FITC/propidium iodide (PI) apoptosis detection kit (Beyotime Biotech, Jiangsu, China) following the manufacturer's instructions. Briefly, the cells were re-suspended and mixed in $500 \mu \mathrm{L}$ binding buffer containing $5 \mu \mathrm{L}$ Annexin 


\section{Cellular Physiology Cell Physiol Biochem 2018;48:1245-1258 \\ \begin{tabular}{l|l} 
DOI: 10.1159/000492010 & O 2018 The Author(s). Published by S. Karger AG, Basel \\
www.karger.com/cpb
\end{tabular}}

Yan et al.: MiR-135a Reduces Lung Injury

V-FITC and $5 \mu \mathrm{L}$ PI. The mixture was incubated in the dark for $15 \mathrm{~min}$ at room temperature. Detection of cell apoptosis was performed by flow cytometry (Becton Dickinson, Franklin Lakes, NJ, USA) and data were analyzed with CellQuest software (Becton Dickinson).

Measurements of intracellular reactive oxygen species (ROS)

The intracellular ROS level was measured as a fluorescent product formed via the oxidation of DCFH-DA (Beyotime Biotech). Briefly, HUVECs were incubated with DCFH-DA $(10 \mu \mathrm{mol} / \mathrm{L})$ at $37^{\circ} \mathrm{C}$ for $30 \mathrm{~min}$. DCFHDA fluorescence intensity was detected at an excitation wavelength of $480 \mathrm{~nm}$ and emission wavelength of $525 \mathrm{~nm}$ by flow cytometry (Becton Dickinson) [19].

\section{Detection of inflammation-related factors by ELISA}

The supernatant was collected by centrifugation at $2000 \mathrm{rpm}$ for $10 \mathrm{~min}$, and tumor necrosis factor- $\alpha$ (TNF- $\alpha$ ), interleukin (IL)-6, and IL-1 $\beta$ were detected according to the manufacturer's instructions (R\&D Systems, Minneapolis, MN, USA). Next, $2 \mu \mathrm{g} / \mathrm{mL}$ monoclonal anti-human mouse primary antibody against TNF- $\alpha$, IL-6, or IL-1 $\beta$, and the corresponding secondary antibodies conjugated to horseradish peroxidase (HRP) were used. Optical density (OD) was then measured in a microplate reader (MK3, Waltham, MA, USA) at a wavelength of $450 \mathrm{~nm}$ [20]. OD from the wells with blank sample (without primary antibody) was subtracted from each of the corresponding treatment samples.

\section{Western blotting}

HUVECs were homogenized in RIPA buffer (Beyotime). Proteins were extracted according to the manufacturer's instruction (Beyotime Biotech). After protein quantification, proteins ( $50 \mu \mathrm{g}$ ) were separated using 10\% sodium dodecyl sulfate polyacrylamide gel electrophoresis gels (Mini-PROTEAN@ 3 Dodeca ${ }^{\mathrm{TM}}$ Cell, Bio-Rad Laboratories) and subsequently transferred onto polyvinylidene fluoride membranes [21]. After blocking, the membranes were incubated with primary antibody against PI3K (1:1000; Ab189403, Abcam, Cambridge, MA, USA), p-PI3K (1:800; Ab182651, Abcam), Akt (1:1000; \#9272, Cell Signaling Technology [CST], Danvers, MA, USA), p-Akt (1:1000; \#9271, CST), Caspase-3 (1:500; Ab44976, Abcam), Bax (1:300; Sc-493, Santa Cruz Biotechnology, Dallas, TX, USA), Bcl2 (1:300; Sc-492, Santa Cruz Biotechnology), PHLPP2 (1:5000; Ab71973, Abcam), or GAPDH (1:2000; \#5174, CST) at $4^{\circ} \mathrm{C}$ overnight, followed by incubation with goat anti-rabbit, donkey anti-goat, or goat anti-mouse HRP-conjugated secondary antibody (1:1000; Beyotime Biotech) for $2 \mathrm{~h}$ at room temperature. Immunoreactive proteins were visualized using the enhanced chemiluminescence Western blotting detection system (Millipore, Billerica, MA, USA) as described previously $[22,23]$. Staining intensity of the bands was measured using a gel image analysis system (Tanon-5200, Shanghai, China).

\section{Dual-luciferase reporter assay}

A dual-luciferase reporter assay was used to investigate the interaction between PHLPP2 and miR-135a. The human PHLPP2 sequence was obtained from PUBMED, and analysis showed an miR-135a binding site in the 3' untranslated region (UTR) of PHLPP2 sequence (AAGCCAU). Then, the 3' UTR of PHLPP2 sequence was introduced to the site following Luc in the pGL3 luciferase vector (Promega, Madison, WI, USA). The sequence was identical to the target sequence, suggesting successful construction of the vector. Next, the single-cell suspension was added to 6 -well plates at a density of $3 \times 10^{5}$ cells/well, followed by incubation at $37^{\circ} \mathrm{C}$ for $24 \mathrm{~h}$. Cells were transfected in the presence of Lipofectamine ${ }^{2} 2000$ as mentioned above (NC, miR$135 \mathrm{a}$ mimic and miR-135a inhibitor). Cells in each group were then treated with PHLPP2-UTR vector (1.5 $\mu \mathrm{g}$ in $250 \mu \mathrm{L}$ serum-free Opti-mem) or blank vector. After transfection for $6 \mathrm{~h}$, the medium was refreshed with complete medium, followed by incubation for another $42 \mathrm{~h}$. The medium was then removed, and cells were washed with phosphate-buffered saline (PBS). After addition of $500 \mu \mathrm{L}$ passive lysis buffer (PLB, Promega), cells were incubated at room temperature for $15 \mathrm{~min}$. Then, $100 \mu \mathrm{L}$ LARII and $20 \mu \mathrm{L}$ lysed cell suspension were added to each well of a 96-well plate, and firefly luciferase activity was measured. After addition of 10 $\mu \mathrm{L}$ Stop\&Glo, bacillus luciferase activity was measured. The ratio of firefly luciferase to bacillus luciferase activity was calculated. 
Determination of barrier integrity by measuring cell permeability

HUVECs were divided into the above-mentioned 7 groups (blank control, NC, NC+MS, mi-miR-135a, mi-miR-135a+MS, si-miR-135a, and si-miR-135a+MS groups). Barrier integrity was determined by measuring cell permeability as described previously [24]. Briefly, at $24 \mathrm{~h}$ after transfection, the cells were seeded and grown to $95-100 \%$ confluence on collagen-coated 6-well BioFlex culture plates. Then, 4 kDa FITC-dextran $(0.4 \mathrm{mg} / \mathrm{mL})$ was directly added into the cell medium. Cells were subjected to MS-induced stress using the Flexcell FX-5000 tension system for 4 $\mathrm{h}$ or $8 \mathrm{~h}$. The plate was then washed 3 times with PBS, and cells were lysed with 5\% Triton X-100. Fluorescence intensity in the cell samples, which reflects the uptake of FITC-dextran, was measured with an Infinite M200 microplate reader (Tecan, Durham, NC, USA).

\section{Statistical analysis}

Statistical analysis was performed with SPSS version 20.0 for Windows. Data are expressed as the mean \pm standard deviation. Comparisons were performed with one-way analysis of variance among groups, followed by post hoc analysis with Tukey's test. A value of $\mathrm{P}<0.05$ was considered statistically significant.

\section{Results}

\section{Efficiency of silencing and over-expression}

To investigate the role of miR-135a in the MSinduced injury in HUVECs, miR-135a was overexpressed or silenced in HUVECs which were then subjected to MS. miR-135a expression in these cells was detected by PCR to evaluate the efficiency of silencing and over-expression. We found that miR-135a expression after miR-135a silencing was reduced to about $1 / 4$ of that in blank control group and NC group $(\mathrm{P}<0.01)$, but increased significantly after over-expression $(\mathrm{P}<0.01)$ as compared with the blank and NC groups (Fig. 1 A1, A2). Protein and mRNA expression of PHLPP2 were detected after silencing or over-expression of miR-135a to investigate whether PHLPP2 was a target of miR-135a. miR-135a over-expression significantly reduced protein and mRNA expression of PHLPP2, but miR-135a silencing markedly increased PHLPP2 expression at the protein and mRNA levels (Fig. 1 B1, B2, B3).

To investigate whether PHLPP2 mediates the effects of miR-135a, PHLPP2 was silenced in HUVECs and PHLPP2 expression was detected at the mRNA and protein levels. mRNA and protein expression were reduced significantly after PHLPP2 silencing as compared with the blank and NC groups (P < 0.01) (Fig. 1 C1, C2, C3).

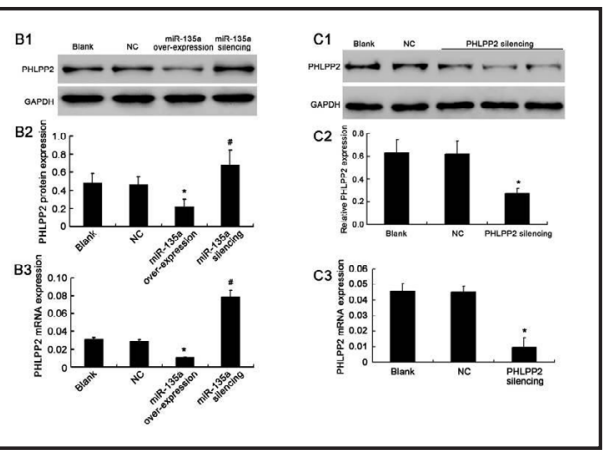

A

B

Fig. 2. Cell density after various treatmentsHUVECs underwent miR-135a silencing or over-expression, followed by MS for $4 \mathrm{~h}$. Cells were observed under light microscopy, and counted in at least 3 fields for each group. ${ }^{*} \mathrm{P}<0.05$ vs blank and NC groups; " $\mathrm{P}<0.05$ vs si-miR$135 \mathrm{a}+\mathrm{MS}$ and $\mathrm{NC}+\mathrm{MS}$ groups. 
Cell density

After culture for $24 \mathrm{~h}$, the HUVECs were spindle-shaped and nearly $100 \%$ confluence was obtained (Fig. 2). In the mi-miR-135a group, the cell density remained unchanged. However, in the si-miR-135a group, the cell density decreased significantly as compared with the blank and NC groups. In addition, after MS, the cell density decreased dramatically in the NC+MS, mi-miR-135a+MS, and si-miR-135a+MS groups, as compared with the corresponding untreated groups, and the cell number in the mi-miR-135a+MS group was markedly higher than in si-miR-135a+MS and NC+MS groups $(\mathrm{P}<0.05)$.

\section{Cell apoptosis}

There is evidence that cyclic MS is able to induce apoptosis of alveolar type II cells [25]. Cell apoptosis was also evaluated after MS in the present study. As expected, MS significantly increased the number of HUVECs in early and later phases of apoptosis as compared with the corresponding untreated groups $(\mathrm{P}<0.05)$. Moreover, silencing of miR-135a alone significantly increased the number of apoptotic cells as compared with the NC and blank groups ( $\mathrm{P}<0.05$ ) (Fig. $3 \mathrm{~A}, \mathrm{~B}$ ). The number of apoptotic cells in the mi-miR-135a+MS group was markedly lower than in the $\mathrm{NC}+\mathrm{MS}$ and si-miR-135a+MS groups $(\mathrm{P}<0.01)$, and a significant difference was also observed between the NC+MS and si-miR-135a+MS groups $(\mathrm{P}<0.05)$ (Fig. 3 A, B). Of note, silencing of miR-135a alone also dramatically increased the number of apoptotic HUVECs as compared with the NC and blank groups $(\mathrm{P}<0.05)$ (Fig. 3 A, B).

We also determined Bax, Bcl-2 and caspase protein expression in HUVECs of the different groups. Our results showed that silencing of miR-135a significantly increased Bax and caspase-3 expression but reduced Bcl-2 expression as compared with the NC and blank groups $(\mathrm{P}<0.05)$ (Fig. 4 A, B). As expected, MS significantly increased Bax and caspase-3 expression and markedly reduced Bcl-2 expression in HUVECs as compared with the corresponding

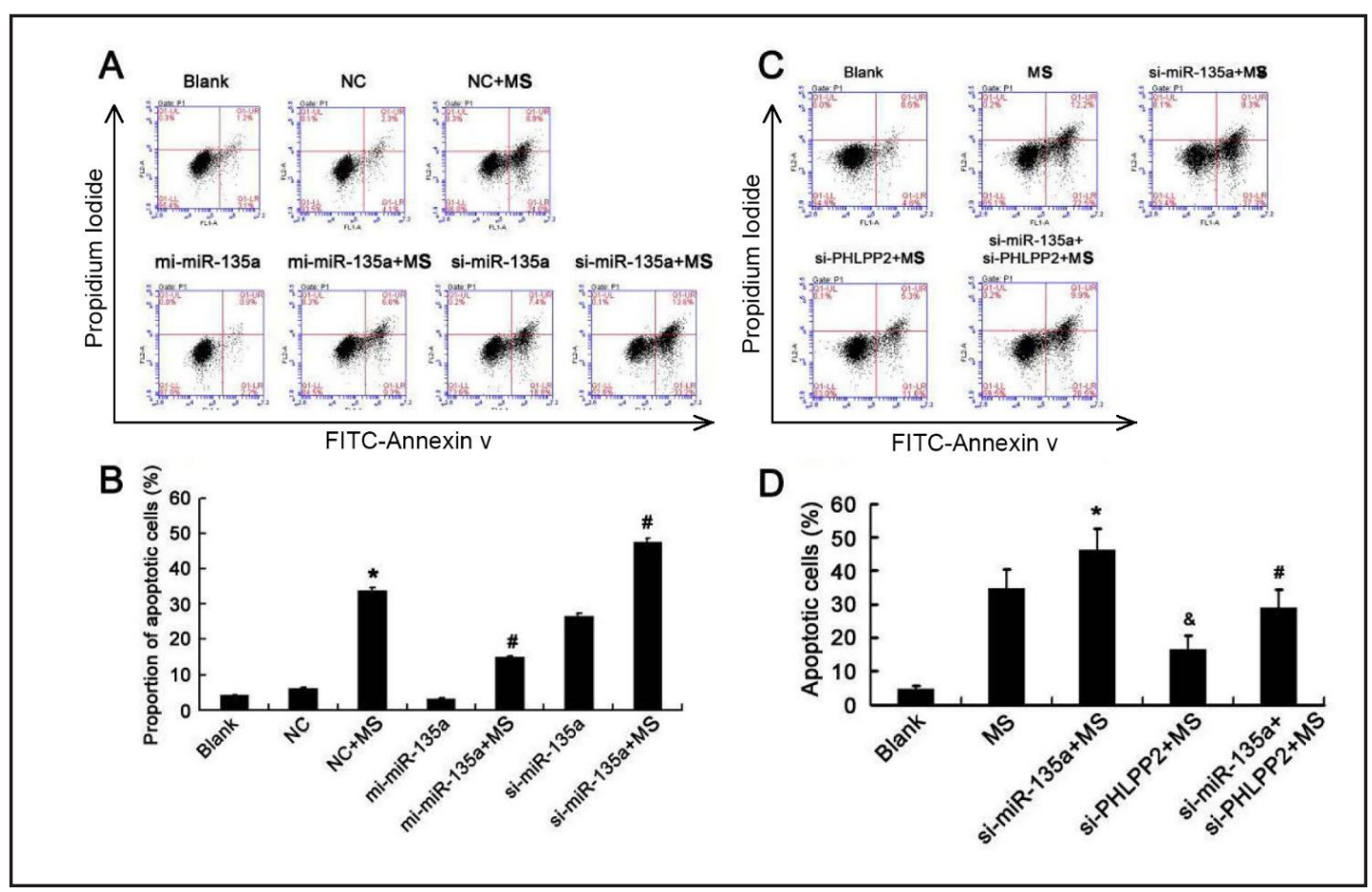

Fig. 3. Cell apoptosis after various treatments HUVECs underwent miR-135a silencing, miR-135a overexpression (A, B) or PHLPP2 silencing (C, D), followed by MS for 4 h. Cells were harvested for the detection of apoptotic cells by flow cytometry. $\mathrm{B}$ : ${ }^{*} \mathrm{P}<0.05$ vs blank and NS groups; ${ }^{\mathrm{P}}<0.05$ vs $\mathrm{NC}+\mathrm{MS}$. $\mathrm{D}$ : ${ }^{*} \mathrm{P}<0.05$ vs blank and MS groups; ${ }^{\&} \mathrm{P}<0.05$ vs MS; " $\mathrm{P}<0.05$ vs si-miR-135a. 
untreated groups (P $<0.05$ ) (Fig. 4 A, B). Furthermore, Bax and caspase- 3 expression in the mi-miR-135a+MS group were markedly lower than in the $\mathrm{NC}+\mathrm{MS}$ and simiR-135a+MS groups, but Bcl-2 expression in the simiR-135a+MS group was significantly higher than in latter two groups $(\mathrm{P}<0.05)$ (Fig. 4 A, B). These findings were consistent with the increase in the number of apoptotic cells after MS.

In addition, apoptotic cells were detected after silencing of PHLPP2 and MS. MS also increased the number of apoptotic cells in HUVECs with PHLPP2 silencing, but the number of apoptotic cells was significantly lower than in the MS group $(\mathrm{P}<0.01$; Fig. $3 \mathrm{C}, \mathrm{D})$. Moreover, in the presence of simultaneous silencing of miR-135a and PHLPP2, the number of apoptotic cells after MS was markedly higher than after silencing of PHLPP2, but significantly lower than after silencing of miR-135a ( $\mathrm{P}<0.05$; Fig. $3 \mathrm{C}, \mathrm{D})$. Western blotting also showed that silencing

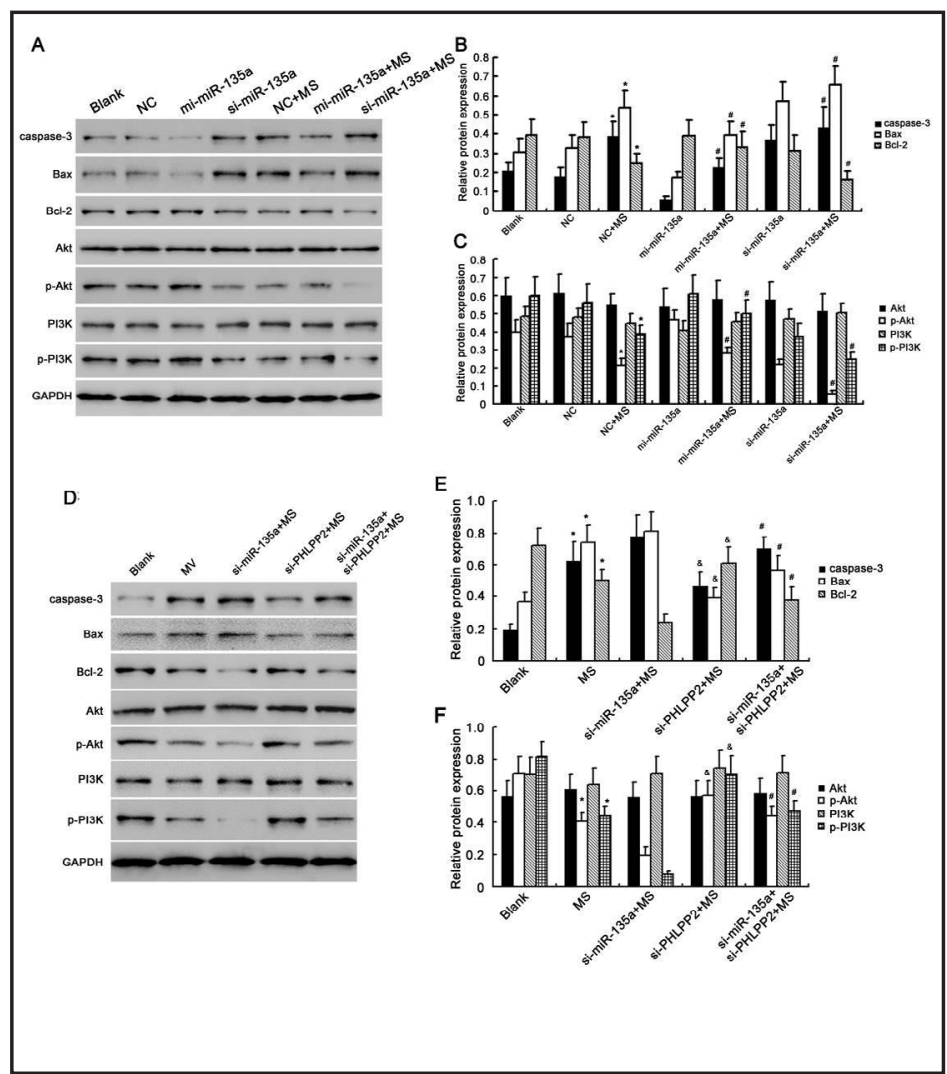

Fig. 4. Expression of apoptosis-related proteins and molecules in the PI3K/Akt pathwayHUVECs underwent miR-135a silencing, miR135a over-expression and PHLPP2 silencing, followed by MS for $4 \mathrm{~h}$. Protein expression of caspase-2, Bcl-2, Bax (A, B), Akt, PI3K, p-Akt, and p-PI3K (A, C) was detected after extraction of total protein by western blotting. GAPDH served as an internal reference. HUVECs underwent PHLPP2 silencing, followed by MS for $4 \mathrm{~h}$ (D, E, F). Protein expression of caspase-2, Bcl-2, Bax (D, E), Akt, PI3K, p-Akt, and p-PI3K (D, F) was detected after extraction of total protein by western blotting. $\mathrm{B}, \mathrm{C}$ : ${ }^{*} \mathrm{P}<0.05$ vs blank and NS groups; ${ }^{*} \mathrm{P}<0.05$ vs $\mathrm{NC}+\mathrm{MS}$ group. $\mathrm{E}$, $\mathrm{F}:{ }^{*} \mathrm{P}<0.05$ vs blank group; ${ }^{\&} \mathrm{P}<0.05$ vs $\mathrm{MS}$ group; ${ }^{*} \mathrm{P}<0.05$ vs si-miR$135 \mathrm{a}+\mathrm{MS}$ group. of PHLPP2 reduced the expression of Bax and caspase-3, but increased Bcl-2 expression in HUVECs after MS as compared with the MS and si-miR-135a+MS groups ( $\mathrm{P}<0.05$ ) (Fig. 4 D, E). After silencing of both miR-135a and PHLPP2, protein expression of Bax and caspase- 3 were lower than in the si-miR-135a+MS group but higher than in the si-PHLPP2+MS group ( $<0.05)$ (Fig. 4 D, E).

\section{Expression of PI3K, $p$-PI3K, Akt, and $p$-Akt}

There is evidence that the PI3K/Akt signaling pathway is involved in the development of VILI [26] and Akt is also a target of PHLPP2 [27]. In the present study, we further detected the protein expression of PI3k and Akt in HUVECs after MS. Our results show that MS significantly inhibited the activation of the PI3K/Akt signaling pathway (reduced expression of p-PI3K and p-Akt, vs blank and NC groups) and silencing of miR-135a further decreased the expression of $\mathrm{p}-\mathrm{PI} 3 \mathrm{~K}$ and p-Akt $(\mathrm{P}<0.05$, si-miR-135a+MS vs NC+MS group) (Fig. $4 \mathrm{~A}$, $\mathrm{C})$. However, miR-135a over-expression increased the expression of $\mathrm{p}$-PI3K and p-Akt as compared with the NC+MS $(\mathrm{P}<0.05)$ and si-miR-135a+MS groups $(\mathrm{P}<0.01)$ (Fig. $4 \mathrm{~A}, \mathrm{C})$. 
Of note, silencing of miR-135a alone also markedly inhibited the activation of the PI3K/Akt pathway (P< 0.05) (Fig. 4 A, C).

In addition, silencing of PHLPP2 increased the protein expression of p-Akt and p-PI3K in HUVECs after MS as compared with the MS group (Fig. 4 D, F). In the presence of silencing of both PHLPP2 and miR-135a, p-Akt and p-PI3K protein expression in HUVECs after MS increased significantly as compared with the si-miR135a+MS group, but decreased significantly as compared with the siPHLPP2+MS group ( $<$ 0.05) (Fig. 4D, F).

\section{ROS}

There is evidence that MV may cause oxidative stress in lung cells [28]. In our study, in vitro MS also significantly increased ROS production in HUVECs as compared with the corresponding untreated groups $(\mathrm{P}<0.05)$ (Fig. 5). In addition, ROS in the mi-miR-135a+MS group were markedly lower than in the $\mathrm{NC}+\mathrm{MS}$ and si-miR$135 \mathrm{a}+\mathrm{MS}$ groups $(\mathrm{P}<0.01)$, and there was a marked difference between the NC+MS and si-miR-135a+MS groups ( $\mathrm{P}<0.05$ ) (Fig. 5). Furthermore, silencing of miR-135a alone also dramatically increased the production of ROS in HUVECs as compared with the NC and blank control groups $(\mathrm{P}<0.05)$ (Fig. 5).

\section{Barrier integrity}

We measured the influence of miR$135 \mathrm{a}$ on MS-induced barrier integrity in HUVECs using the FITC-dextran assay. MS for $4 \mathrm{~h}$ induced obvious barrier dysfunction (Fig. $6 \mathrm{~A}$ ). Moreover, MS for $8 \mathrm{~h}$ induced greater reduction of barrier integrity compared with treatment for 4 h (Fig. 6 B). Notably, administration of an miR-135a mimic significantly reduced the MS-induced barrier dysfunction in both experiments ( $4 \mathrm{~h}$ and $8 \mathrm{~h}$ ) (Fig. $6 \mathrm{~A}, \mathrm{~B}$ ). By contrast, knockdown of miR-135a further decreased barrier dysfunction induced by MS (Fig. $6 \mathrm{~A}$, B).

\section{Inflammatory cytokines}

Studies have shown that MV may induce inflammation in lung cells [29, 30]. In our study, inflammatory cytokines (TNF- $\alpha$, IL-1 $\beta$, and IL-6) were detected in HUVECs after in vitro MS. As shown in Fig.

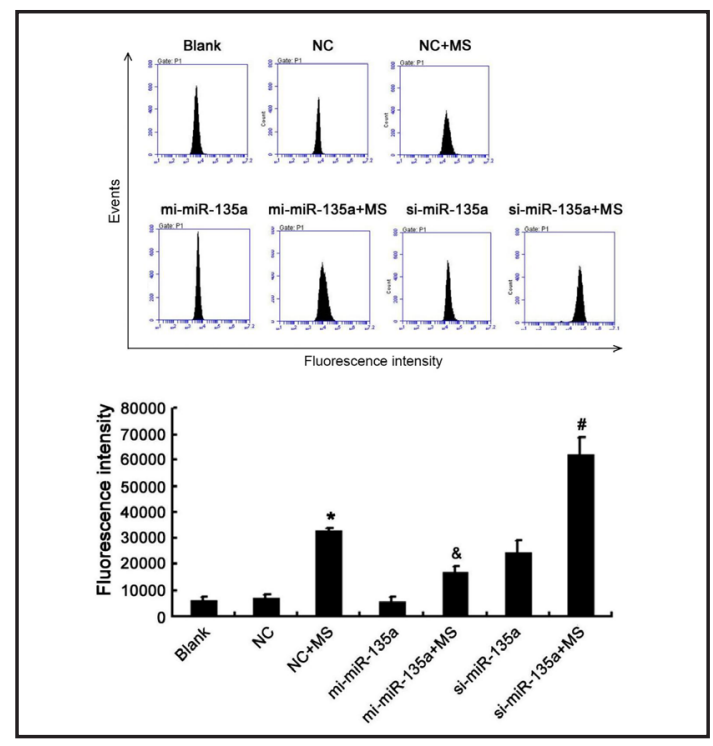

Fig. 5. Intracellular ROS content in HUVECs after various treatments HUVECs underwent miR-135a silencing or over-expression, followed by MS for $4 \mathrm{~h}$. Cells were then harvested for the detection of intracellular ROS by flow cytometry. ${ }^{*} \mathrm{P}<0.05$ vs blank and NS groups; ${ }^{\circledR} \mathrm{P}<0.05$, mi-miR-135a+MS vs $\mathrm{NC}+\mathrm{MS}$; ${ }^{\mathrm{P}}<0.05$, si-miR-135a+MS vs NC+MS.

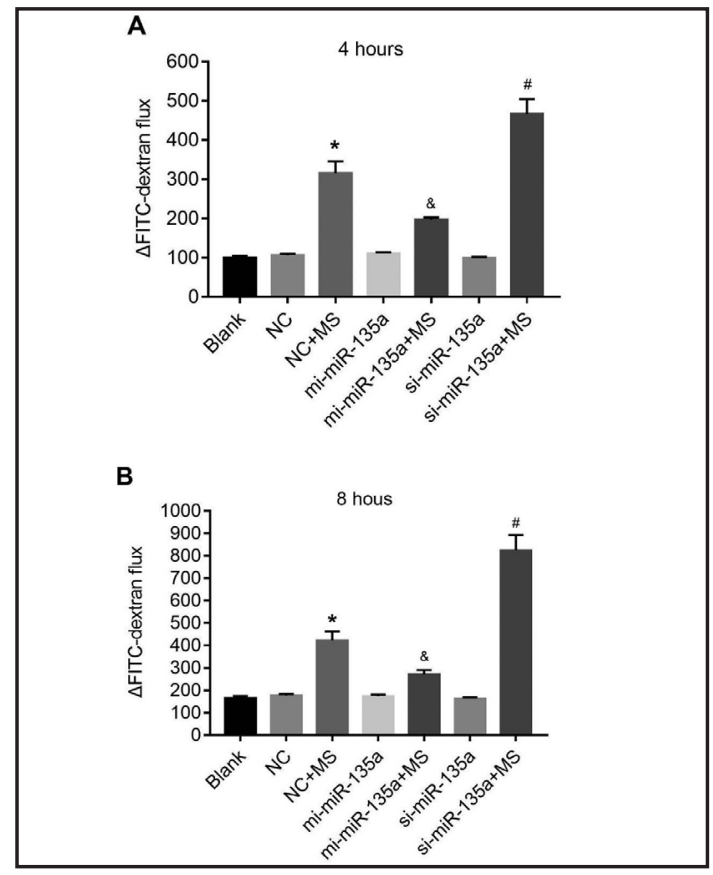

Fig. 6. Barrier integrity of HUVECs after various treatments HUVECs underwent miR-135a silencing or over-expression, followed by MS for $4 \mathrm{~h}$ (A) and $8 \mathrm{~h}$ (B). Cells were then subjected to FITC-dextran assay to determine barrier function. ${ }^{*} \mathrm{P}<0.05$ vs blank and NS groups; ${ }^{\mathrm{P}}<0.05$, mi-miR-135a+MS vs NC+MS; ${ }^{P}<0.05$, si-miR-135a+MS vs NC+MS. 
7, in vitro MS significantly increased these inflammatory cytokines as compared with the corresponding untreated groups $(\mathrm{P}<$ $0.05)$. In addition, the levels of inflammatory cytokines secreted by HUVECs in the mimiR-135a+MS group were markedly lower than in the NC+MS and si-miR-135a+MS groups $(\mathrm{P}<0.05)$ and there was a marked difference between the $\mathrm{NC}+\mathrm{MS}$ and simiR-135a+MS groups $(P<0.05)$ (Fig. 7). Of note, silencing of miR-135a alone also dramatically increased the levels of these inflammatory cytokines in HUVECs as compared with the $\mathrm{NC}$ and blank control groups $(\mathrm{P}<0.05)$ (Fig. 7).

\section{Dual-luciferase reporter assay}

In the presence of blank pGL3 luciferase vector, the luciferase activity ratio was comparable among the three groups $(\mathrm{P}>0.05$; Fig. 8 A). However, after introduction of 3' UTR of PHLPP2 into this vector, miR-135a mimic significantly reduced the luciferase activity ratio, but miR-135a inhibitor markedly increased the luciferase activity ratio as compared with the NC group (Fig. 8 B). This suggests that miR-135a is able to interact with PHLPP2.

\section{Discussion}

In this study, we investigated the effects of miR-135a on MS-induced cell injury. We found that (1) MS increased apoptosis in HUVECs, production of inflammatory cytokines and generation of ROS in vitro; (2) silencing of miR-135a decreased MSinduced injury of HUVECs, whereas miR135 a over-expression had the opposite effect; and (3) the protective effects of miR-135a may be related to its inhibition of PHLPP2 and subsequent activation of the PI3K/Akt signaling pathway.

A number of molecular mechanisms may contribute to acute lung injury [3134]. To date, miRNA profile has been the focus in biological studies related to acute lung injury. Although the role of miR-135a has been extensively investigated in cancer, little is known about its role in VILI. In this study, we investigated the role of miR-135a in VILI in vitro by focusing on inflammation, oxidative stress, and apoptosis, which are

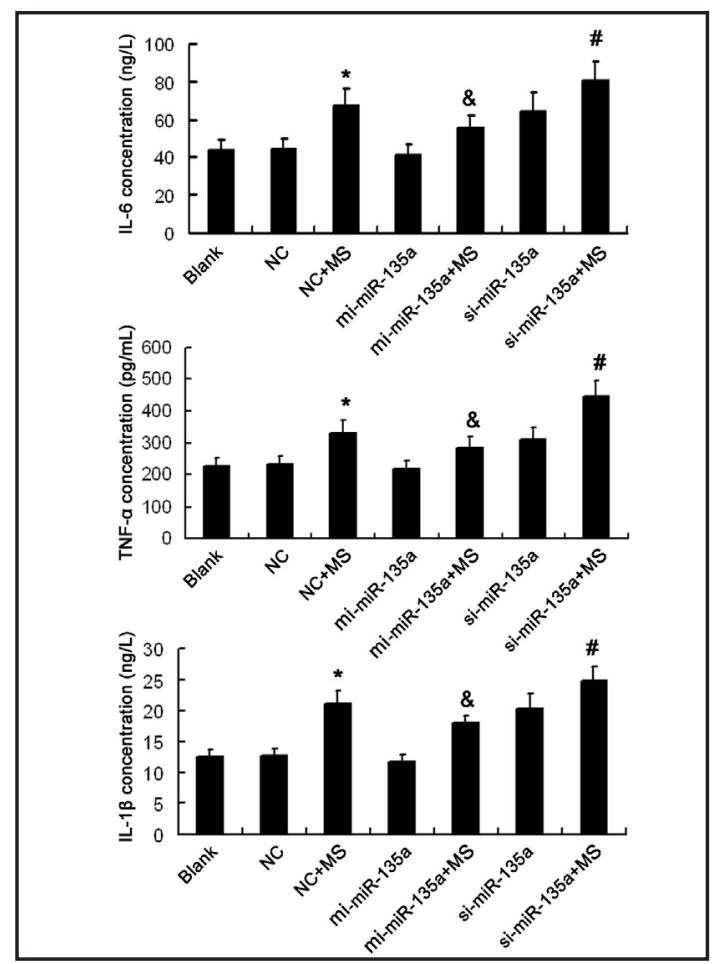

Fig. 7. Inflammatory cytokines in HUVECs after various treatments HUVECs underwent miR-135a silencing or over-expression, followed by MS for $4 \mathrm{~h}$. Cells were then harvested for the detection of intracellular ROS by ELISA. ${ }^{*} \mathrm{P}<0.05$ vs blank and NS groups; ${ }^{\&} \mathrm{P}<0.05$, mi-miR-135a+MS vs NC+MS; ${ }^{\#} \mathrm{P}<0.05$, si-miR-135a+MS vs NC+MS.

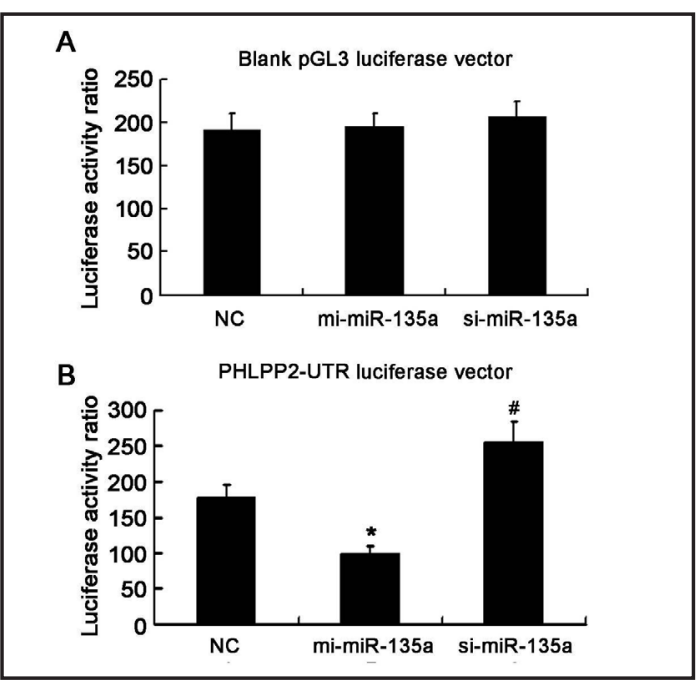

Fig. 8. Dual-luciferase reporter assay of HUVECs HUVECs were treated with blank pGL3 luciferase vector (A) or PHLPP2-UTR luciferase vector (B), followed by incubation with an miR-135a mimic or inhibitor. The luciferase activity was detected. ${ }^{*} \mathrm{P}<0.05$ vs $\mathrm{NC}$ group; ${ }^{\#} \mathrm{P}<0.05$ vs $\mathrm{NC}$ group. 
three critical molecular mechanisms involved in the pathogenesis of VILI. It is clear that knockdown of miR-135a significantly increased apoptosis under MS-induced stress (Fig. 3 ). This result was supported by data showing significant upregulation of pro-apoptotic factors Bax and caspase-3 (Fig. 4) in cells with miR-135a knockdown; overexpression of miR-135a had the opposite effect. To provide more evidence about function of miR135a, we measured intracellular ROS content (Fig. 5), barrier integrity (Fig. 6), and the levels of three pro-inflammatory cytokines (TNF- $\alpha$, IL-6, and IL-1 $\beta$ ) (Fig. 7). Under MSinduced stress, knockdown of miR-135a further triggered an increase in ROS content and enhanced concentrations of TNF- $\alpha$, IL-6, and IL-1 $\beta$, whereas overexpression of miR-135a suppressed ROS content and decreased TNF- $\alpha$, IL- 6 , and IL- $1 \beta$ levels. Based on these results, we considered that miR-135a protects against MS-induced stress in cells. Of note, it has been shown that inhibition of miR-135a was able to protect A549 cells from LPS-induced apoptosis by targeting Bcl-2 [35] and over-expression of miR-135a blocked Bcl-2 protein and enhanced $\mathrm{H}_{2} \mathrm{O}_{2}$-induced apoptosis in $\mathrm{H} 9 \mathrm{c} 2$ cells [36]; upregulation of miR-135a could aggravate sepsis-induced inflammation in BALB/c mice [11]. These discrepancies might be attributed to differences in cell types.

PHLPPs are a class of potent inhibitor of Aky that dephosphorylate Akt isoforms at Ser473 and have been intensively investigated in cancer. Due to this feature, PHLPPs critically regulate cellular homeostasis, and the dysregulation of PHLPPs is tightly associated with various pathophysiological changes [37]. In this study, we found that PHLPP2 silencing markedly inhibited MS-induced apoptosis in HUVECs. Findings from the dual-luciferase reporter assay confirmed that miR-135a could interact with PHLPP2 (Fig. 8). These results indicate that miR-135a suppressed PHLPP2 protein level in HUVECs under MS-induced stress. Since PHLPP2 dephosphorylates Akt at Ser473, the reduced PHLPP2 in miR-135aoverexpressed HUVECs would activate the Akt signaling pathway. It should be noted that the Akt pathway is a classical pro-survival signaling pathway in cells. Thus, we believe that the pro-survival action of miR-135a is at least partially mediated by PHLPP2.

Interestingly, PHLPP2 participates in the biological functions of many miRNAs. In a previous study, Mao et al. found that miR-135a enhanced cellular proliferation through targeting PHLPP2 and FOXO1 in human bladder cancer [10]. Moreover, PHLPP2 seemed to be a crucial mediator of the regulatory effects of miR-32 [38], miR-760 [39], miR-181a [40], miR27a [41], and miR-938 [42] on cell proliferation and migration. Our results provide the first evidence that PHLPP2 may be the direct target of miR-135a and contributes to the influence of miR-135a on HUVEC survival under MS-induced stress. However, it is unlikely that a single phosphatase PHLPP2 could be responsible for all the endothelial derangements associated with VILI. In this case, the miR-135a-PHLPP2 pathway may be only one of several regulatory mechanisms involved in VILI-associated endothelial dysfunction. Further investigations focusing on the roles of miRNAs and their targets in VILI-associated endothelial dysfunction are warranted.

The preservation of vascular endothelial cell barrier integrity is critical to vessel homeostasis, and barrier dysfunction is a key feature of acute lung injury [43] and VILI [6]. The reduced endothelial barrier integrity in VILI is due to mechanical ventilation at high tidal volume and the associated pulmonary capillary leakage and acute lung inflammation. SC5b9, a measure of complement activation, causes an increase in rat pulmonary microvascular permeability by inducing activation of RhoA and subsequent phosphorylation of myosin light chain and contraction of endothelial cells [44]. Caveolin-1, a key regulator of pulmonary endothelial barrier function, is required for MS-induced pulmonary edema and endothelial hyperpermeability in vitro and in vivo [45]. To explore the role of miR-135a in pulmonary alveolar barrier function, we further studied the effect of miR-135a overexpression and knockdown on endothelial barrier properties in vitro. As expected, our results clearly showed that miR-135a protected against MS-induced barrier dysfunction (Fig. 6). We believe that this protective effect of miR-135a on endothelial barrier integrity may contribute to the beneficial actions of miR-135a in VILI. 
There is evidence that Akt, PKC, the Ras-Raf-MEK-ERK pathway, AGC kinase family members, and other molecules may be the targets of PHLPPs [37]. In addition, studies indicate that PHLPP1 and PHLPP2 may both serve as tumor suppressors, largely attributed to PHLPP-dependent suppression of pro-survival signaling, such as through the PI3K/ Akt signaling pathway [37, 46]. The phosphatidylinositol-3 kinase (PI3K) signaling pathway plays an essential role in some physiological and cellular processes including cell proliferation, growth, survival, migration, and metabolism [47]. Studies have shown that the PI3K signaling pathway is involved in the pathogenesis of some respiratory diseases and may serve as a target in the treatment of these diseases [47]. The role of the PI3K pathway in the pathogenesis of VILI is still controversial [48]. Shu et al. found that an inhibitor of IкB kinase (IKK16) may attenuate VILI via activation of the PI3K/Akt signaling pathway in Sprague-Dawley rats [49]. In a study by Faller et al., results showed that protection of isoflurane during MV was accompanied by substantial phosphorylation of Akt protein and inhibition of PI3K/Akt signaling before MV completely reversed the lung-protective effects of isoflurane treatment in C57BL/6N mice [50]. However, in earlier studies, results indicated that PI3K- $\gamma$ in resident lung cells mediated part of the alveolar edema induced by high-stress ventilation [51] and silencing of PI3K- $\gamma$ seemed to attenuate functional and morphological consequences of VILI [52]. In addition, pretreatment with a PI3K inhibitor (LY-294002) significantly attenuated VILI, whereas an Akt inhibitor markedly reduced VILI in isolated lungs [53]. Thus, we further investigated the expression of target molecules of PHLPP2 in the PI3K/Akt signaling pathway. Our results showed that MS could inhibit the PI3K/ Akt signaling pathway (reduction in p-PI3K and p-Akt expression) and silencing of miR135a further reduced p-PI3K and p-Akt expression in HUVECs. However, miR-135a overexpression significantly activated the PI3K/Akt signaling pathway (characterized by marked increase in p-PI3K and p-Akt expression) (Fig. 4), which was accompanied by improvement of HUVEC injury. Furthermore, silencing of PHLPP2 increased p-Akt and p-PI3K protein expression, but simultaneous silencing of miR-135a compromised activation of the PI3K/ Akt signaling pathway after PHLPP2 silencing in HUVECs (Fig. 4).

Several possible limitations of this study should be considered. After PHLPP2 silencing, we detected apoptotic cells following MS, but whether PHLPP2 silencing alone affects the apoptosis of HUVECs was not investigated, and other parameters were not measured after PHLPP2 silencing in HUVECs following MS. In addition, this study was conducted in HUVECs, and pulmonary endothelial cells are more representative of cells involved in VILI $[54,55]$. Thus, our results need to be confirmed in pulmonary endothelial cells and in animal models in the future.

Taken together, our findings suggest that miR-135a may inhibit PHLPP2 to activate the PI3K/Akt signaling pathway, exerting protective effects on MS-induced injury to HUVECs.

\section{Acknowledgement}

This study was supported by grants from the National Science Foundation of China (No. 81671304), the Shanghai Natural Science Foundation (No. 15ZR1414200), and the Nursery Program of the Second Military Medical University (No. 2014QN16).

\section{Disclosure Statement}

The authors declare that there are no conflicts of interest related to this study.

\section{References}

Beitler JR, Malhotra A, Thompson BT: Ventilator-induced Lung Injury. Clin Chest Med 2016;37:633-646.

Ware LB, Matthay MA: The acute respiratory distress syndrome. N Engl J Med 2000;342:1334-1349. 


\section{Cellular Physiology Cell Physiol Biochem 2018;48:1245-1258 \begin{tabular}{l|l} 
and Biochemistry Published onlIne: July 25, 2018 & $\begin{array}{l}\text { C) } 2018 \text { The Author(s). Published by S. Karger AG, Basel } \\
\text { www.karger.com/cpb }\end{array}$ \\
\hline
\end{tabular}}

-3 Acute Respiratory Distress Syndrome N, Brower RG, Matthay MA, Morris A, Schoenfeld D, Thompson BT, Wheeler A: Ventilation with lower tidal volumes as compared with traditional tidal volumes for acute lung injury and the acute respiratory distress syndrome. N Engl J Med 2000;342:1301-1308.

4 Kuipers MT, van der Poll T, Schultz MJ, Wieland CW: Bench-to-bedside review: Damage-associated molecular patterns in the onset of ventilator-induced lung injury. Crit Care 2011;15:235.

5 Lin HJ, Hsu CC, Chio CC, Tian YF, Lin MT, Lin TW, Chang CH, Chang CP: Gamma-Secretase Inhibitors Attenuate Neurotrauma and Neurogenic Acute Lung Injury in Rats by Rescuing the Accumulation of Hypertrophic Microglia. Cell Physiol Biochem 2017;44:1726-1740.

-6 Wang T, Gross C, Desai AA, Zemskov E, Wu X, Garcia AN, Jacobson JR, Yuan JX, Garcia JG, Black SM: Endothelial cell signaling and ventilator-induced lung injury: molecular mechanisms, genomic analyses, and therapeutic targets. Am J Physiol Lung Cell Mol Physiol 2017;312:L452-L476.

7 Ha M, Kim VN: Regulation of microRNA biogenesis. Nat Rev Mol Cell Biol 2014;15:509-524.

8 Rajasekaran S, Pattarayan D, Rajaguru P, Sudhakar Gandhi PS, Thimmulappa RK: MicroRNA Regulation of Acute Lung Injury and Acute Respiratory Distress Syndrome. J Cell Physiol 2016;231:2097-2106.

-9 Vaporidi K, Vergadi E, Kaniaris E, Hatziapostolou M, Lagoudaki E, Georgopoulos D, Zapol WM, Bloch KD, Iliopoulos D: Pulmonary microRNA profiling in a mouse model of ventilator-induced lung injury. Am J Physiol Lung Cell Mol Physiol 2012;303:L199-207.

10 Mao XP, Zhang LS, Huang B, Zhou SY, Liao J, Chen LW, Qiu SP, Chen JX: Mir-135a enhances cellular proliferation through post-transcriptionally regulating PHLPP2 and FOXO1 in human bladder cancer. J Transl Med 2015;13:86.

11 Zheng G, Pan M, Jin W, Jin G, Huang Y: MicroRNA-135a is up-regulated and aggravates myocardial depression in sepsis via regulating p38 MAPK/NF-kappaB pathway. Int Immunopharmacol 2017;45:6-12.

12 Mannironi C, Biundo A, Rajendran S, De Vito F, Saba L, Caioli S, Zona C, Ciotti T, Caristi S, Perlas E, Del Vecchio G, Bozzoni I, Rinaldi A, Mele A, Presutti C: miR-135a Regulates Synaptic Transmission and AnxietyLike Behavior in Amygdala. Mol Neurobiol 2017;

13 Oliva-Olivera W, Lhamyani S, Coin-Araguez L, Castellano-Castillo D, Alcaide-Torres J, Yubero-Serrano EM, El Bekay R, Tinahones FJ: Neovascular deterioration, impaired NADPH oxidase and inflammatory cytokine expression in adipose-derived multipotent cells from subjects with metabolic syndrome. Metabolism 2017;71:132-143.

14 Moon HS, Batirel S, Mantzoros CS: Alpha linolenic acid and oleic acid additively down-regulate malignant potential and positively cross-regulate AMPK/S6 axis in OE19 and OE33 esophageal cancer cells. Metabolism 2014;63:1447-1454.

15 Gong Y, Ma Y, Ye Z, Fu Z, Yang P, Gao B, Guo W, Hu D, Ye J, Ma S, Zhang F, Zhou L, Xu X, Li Z, Yang T, Zhou H: Thyroid stimulating hormone exhibits the impact on LDLR/LDL-c via up-regulating hepatic PCSK9 expression. Metabolism 2017;76:32-41.

16 Aroor AR, Jia G, Habibi J, Sun Z, Ramirez-Perez FI, Brady B, Chen D, Martinez-Lemus LA, Manrique C, Nistala R, Whaley-Connell AT, Demarco VG, Meininger GA, Sowers JR: Uric acid promotes vascular stiffness, maladaptive inflammatory responses and proteinuria in western diet fed mice. Metabolism 2017;74:32-40.

17 Li DJ, Liu J, Hua X, Fu H, Huang F, Fei YB, Lu WJ, Shen FM, Wang P: Nicotinic acetylcholine receptor alpha7 subunit improves energy homeostasis and inhibits inflammation in nonalcoholic fatty liver disease. Metabolism 2017;

18 Sheikh AQ Kuesel C, Taghian T, Hurley JR, Huang W, Wang Y, Hinton RB, Narmoneva DA: Angiogenic microenvironment augments impaired endothelial responses under diabetic conditions. Am J Physiol Cell Physiol 2014;306:C768-778.

19 Li DJ, Fu H, Zhao T, Ni M, Shen FM: Exercise-stimulated FGF23 promotes exercise performance via controlling the excess reactive oxygen species production and enhancing mitochondrial function in skeletal muscle. Metabolism 2016;65:747-756.

20 Deng F, Wang S, Zhang L, Xie X, Cai S, Li H, Xie GL, Miao HL, Yang C, Liu X, Xia Z: Propofol Through Upregulating Caveolin-3 Attenuates Post-Hypoxic Mitochondrial Damage and Cell Death in H9C2 Cardiomyocytes During Hyperglycemia. Cell Physiol Biochem 2017;44:279-292.

-21 Ito Y, Hsu MF, Bettaieb A, Koike S, Mello A, Calvo-Rubio M, Villalba JM, Haj FG: Protein tyrosine phosphatase 1B deficiency in podocytes mitigates hyperglycemia-induced renal injury. Metabolism 2017;76:56-69. 


\section{Cellular Physiology Cell Physiol Biochem 2018;48:1245-1258 \begin{tabular}{l|l} 
DOI: 10.1159/000492010 & $\begin{array}{l}\text { O 2018 The Author(s). Published by S. Karger AG, Basel } \\
\text { www.karger.com/cpb }\end{array}$
\end{tabular}}

Yan et al.: MiR-135a Reduces Lung Injury

22 Li DJ, Li YH, Yuan HB, Qu LF, Wang P: The novel exercise-induced hormone irisin protects against neuronal injury via activation of the Akt and ERK1/2 signaling pathways and contributes to the neuroprotection of physical exercise in cerebral ischemia. Metabolism 2017;68:31-42.

23 Li DJ, Fu H, Tong J, Li YH, Qu LF, Wang P, Shen FM: Cholinergic anti-inflammatory pathway inhibits neointimal hyperplasia by suppressing inflammation and oxidative stress. Redox Biol 2017;15:22-33.

-24 Slomka N, Gefen A: Relationship Between Strain Levels and Permeability of the Plasma Membrane in Statically Stretched Myoblasts. Annals of Biomedical Engineering 2012;40:606-618.

-25 Hammerschmidt S, Kuhn H, Grasenack T, Gessner C, Wirtz H: Apoptosis and necrosis induced by cyclic mechanical stretching in alveolar type II cells. Am J Respir Cell Mol Biol 2004;30:396-402.

-26 Hammerschmidt S, Kuhn H, Gessner C, Seyfarth HJ, Wirtz H: Stretch-induced alveolar type II cell apoptosis: role of endogenous bradykinin and PI3K-Akt signaling. Am J Respir Cell Mol Biol 2007;37:699-705.

27 Newton AC, Trotman LC: Turning off AKT: PHLPP as a drug target. Annu Rev Pharmacol Toxicol 2014;54:537-558.

28 Tanaka T, Saito Y, Matsuda K, Kamio K, Abe S, Kubota K, Azuma A, Gemma A: Cyclic mechanical stretchinduced oxidative stress occurs via a NOX-dependent mechanism in type II alveolar epithelial cells. Respir Physiol Neurobiol 2017;242:108-116.

29 Held HD, Boettcher S, Hamann L, Uhlig S: Ventilation-induced chemokine and cytokine release is associated with activation of nuclear factor-kappaB and is blocked by steroids. Am J Respir Crit Care Med 2001;163:711-716.

-30 Wang Y, Xu CF, Liu YJ, Mao YF, Lv Z, Li SY, Zhu XY, Jiang L: Salidroside Attenuates Ventilation Induced Lung Injury via SIRT1-Dependent Inhibition of NLRP3 Inflammasome. Cell Physiol Biochem 2017;42:34-43.

-31 Tai W, Xu Y, Ding J, Wu H, Du M, Qu X, Gao L, Li J, Dong Z: Fibrocytes Ameliorate Acute Lung Injury by Decreasing Inflammatory Cytokine and Chemokine Levels and Reducing Neutrophil Accumulation in the Lung. Cell Physiol Biochem 2017;44:1526-1536.

-32 Chen Y, Wang L, Kang Q, Zhang X, Yu G, Wan X, Wang J, Zhu K: Heat Shock Protein A12B Protects Vascular Endothelial Cells Against Sepsis-Induced Acute Lung Injury in Mice. Cell Physiol Biochem 2017;42:156168.

-33 Liu Y, Lu J, Wang X, Chen L, Liu S, Zhang Z, Yao W: Erythropoietin-Derived Peptide Protects Against Acute Lung Injury After Rat Traumatic Brain Injury. Cell Physiol Biochem 2017;41:2037-2044.

34 Xu X, Zhu Q, Zhang R, Wang Y, Niu F, Wang W, Sun D, Wang A: ITRAQ-Based Proteomics Analysis of Acute Lung Injury Induced by Oleic Acid in Mice. Cell Physiol Biochem 2017;44:1949-1964.

- 35 Zhao J, Li X, Zou M, He J, Han Y, Wu D, Yang H, Wu J: miR-135a inhibition protects A549 cells from LPSinduced apoptosis by targeting Bcl-2. Biochem Biophys Res Commun 2014;452:951-957.

- 36 Liu N, Shi YF, Diao HY, Li YX, Cui Y, Song XJ, Tian X, Li TY, Liu B: MicroRNA-135a Regulates Apoptosis Induced by Hydrogen Peroxide in Rat Cardiomyoblast Cells. Int J Biol Sci 2017;13:13-21.

- 37 Brognard J, Newton AC: PHLiPPing the switch on Akt and protein kinase C signaling. Trends Endocrinol Metab 2008;19:223-230.

-38 Xia H, Long J, Zhang R, Yang X, Ma Z: MiR-32 contributed to cell proliferation of human breast cancer cells by suppressing of PHLPP2 expression. Biomed Pharmacother 2015;75:105-110.

39 Liao Y, Deng Y, Liu J, Ye Z, You Z, Yao S, He S: MiR-760 overexpression promotes proliferation in ovarian cancer by downregulation of PHLPP2 expression. Gynecol Oncol 2016;143:655-663.

40 Rang Z, Wang ZY, Pang QY, Wang YW, Yang G, Cui F: MiR-181a Targets PHLPP2 to Augment AKT Signaling and Regulate Proliferation and Apoptosis in Human Keloid Fibroblasts. Cell Physiol Biochem 2016;40:796806.

-41 Ding L, Zhang S, Xu M, Zhang R, Sui P, Yang Q: MicroRNA-27a contributes to the malignant behavior of gastric cancer cells by directly targeting PH domain and leucine-rich repeat protein phosphatase 2. J Exp Clin Cancer Res 2017;36:45.

42 Li CF, Li YC, Jin JP, Yan ZK, Li DD: miR-938 promotes colorectal cancer cell proliferation via targeting tumor suppressor PHLPP2. Eur J Pharmacol 2017;807:168-173.

43 Lucas R, Verin AD, Black SM, Catravas JD: Regulators of endothelial and epithelial barrier integrity and function in acute lung injury. Biochem Pharmacol 2009;77:1763-1772.

44 Liu K, Mao YF, Zheng J, Peng ZY, Liu WW, Liu Y, Xu WG, Sun XJ, Jiang CL, Jiang L: SC5b-9-induced pulmonary microvascular endothelial hyperpermeability participates in ventilator-induced lung injury. Cell Biochem Biophys 2013;67:1421-1431. 


\section{Cellular Physiology Cell Physiol Biochem 2018;48:1245-1258 \begin{tabular}{l|l} 
DOI: 10.1159/000492010 & $\begin{array}{l}\text { O 2018 The Author(s). Published by S. Karger AG, Basel } \\
\text { www.karger.com/cpb }\end{array}$
\end{tabular}}

Yan et al.: MiR-135a Reduces Lung Injury

45 Maniatis NA, Kardara M, Hecimovich D, Letsiou E, Castellon M, Roussos C, Shinin V, Votta-Vellis EG, Schwartz DE, Minshall RD: Role of caveolin-1 expression in the pathogenesis of pulmonary edema in ventilator-induced lung injury. Pulm Circ 2012;2:452-460.

46 Grzechnik AT, Newton AC: PHLPPing through history: a decade in the life of PHLPP phosphatases. Biochem Soc Trans 2016;44:1675-1682.

47 Yuan TL, Cantley LC: PI3K pathway alterations in cancer: variations on a theme. Oncogene 2008;27:54975510 .

48 Uhlig S, Uhlig U: Pharmacological interventions in ventilator-induced lung injury. Trends Pharmacol Sci 2004;25:592-600.

49 Shu YS, Tao W, Miao QB, Zhu YB, Yang YF: Improvement of ventilation-induced lung injury in a rodent model by inhibition of inhibitory kappaB kinase. J Trauma Acute Care Surg 2014;76:1417-1424.

50 Faller S, Strosing KM, Ryter SW, Buerkle H, Loop T, Schmidt R, Hoetzel A: The volatile anesthetic isoflurane prevents ventilator-induced lung injury via phosphoinositide 3-kinase/Akt signaling in mice. Anesth Analg 2012;114:747-756.

51 Fanelli V, Puntorieri V, Assenzio B, Martin EL, Elia V, Bosco M, Delsedime L, Del Sorbo L, Ferrari A, Italiano S, Ghigo A, Slutsky AS, Hirsch E, Ranieri VM: Pulmonary-derived phosphoinositide 3-kinase gamma (PI3Kgamma) contributes to ventilator-induced lung injury and edema. Intensive Care Med 2010;36:19351945.

52 Lionetti V, Lisi A, Patrucco E, De Giuli P, Milazzo MG, Ceci S, Wymann M, Lena A, Gremigni V, Fanelli V, Hirsch E, Ranieri VM: Lack of phosphoinositide 3-kinase-gamma attenuates ventilator-induced lung injury. Crit Care Med 2006;34:134-141.

53 Miyahara T, Hamanaka K, Weber DS, Drake DA, Anghelescu M, Parker JC: Phosphoinositide 3-kinase, Src, and Akt modulate acute ventilation-induced vascular permeability increases in mouse lungs. Am J Physiol Lung Cell Mol Physiol 2007;293:L11-21.

-54 Meliton A, Meng F, Tian Y, Shah AA, Birukova AA, Birukov KG: Role of Krev Interaction Trapped-1 in Prostacyclin-Induced Protection against Lung Vascular Permeability Induced by Excessive Mechanical Forces and Thrombin Receptor Activating Peptide 6. Am J Respir Cell Mol Biol 2015;53:834-843.

55 Birukova AA, Fu P, Xing J, Cokic I, Birukov KG: Lung endothelial barrier protection by iloprost in the 2-hit models of ventilator-induced lung injury (VILI) involves inhibition of Rho signaling. Transl Res 2010;155:44-54. 